\title{
LOCALIZATION, HURWITZ NUMBERS AND THE WITTEN CONJECTURE*
}

\author{
LIN $\mathrm{CHEN}^{\dagger}$, YI $\mathrm{LI}^{\ddagger}$, AND KEFENG LIU $\S$
}

\begin{abstract}
In this note, we use the combinatorial method of Goulden-Jackson-Vakil to give a simple proof of Witten conjecture-Kontsevich theorem.
\end{abstract}

Key words. Moduli space, intersection number.

AMS subject classifications. $14 \mathrm{~N} 35$

1. Introduction. The well-known Witten conjecture states that the intersection theory of the $\psi$ classes on the moduli spaces of Riemann surfaces is equivalent to the "Hermitian matrix model" of two-dimensional gravity. All $\psi$-integrals can be efficiently computed by using the Witten conjecture [13], first proved by Kontsevich [6]. Today, there are many different approach to prove this conjecture, see [4], [5], [11] and [12] . For convenience, we use Witten's natation

$$
\left\langle\tau_{\beta_{1}} \cdots \tau_{\beta_{n}}\right\rangle_{g}:=\int_{\overline{\mathcal{M}}_{g, n}} \psi_{1}^{\beta_{1}} \cdots \psi_{n}^{\beta_{n}} .
$$

The natural generating function for the $\psi$-integrals described above is

$$
F_{g}(t):=\sum_{n \geq 0} \frac{1}{n !} \sum_{k_{1}, \cdots, k_{n}} t_{k_{1}} \cdots t_{k_{n}}\left\langle\tau_{\beta_{1}} \cdots \tau_{\beta_{n}}\right\rangle_{g}, \quad F(t, \lambda):=\sum_{g \geq 0} F_{g} \lambda^{2 g-2} .
$$

For example, the first system of differential equations conjectured by Witten are the KDV equations. Let $F(t):=F(t, 1)$, define

$$
\left\langle\left\langle\tau_{\beta_{1}} \cdots \tau_{\beta_{n}}\right\rangle\right\rangle:=\frac{\partial}{\partial t_{k_{1}}} \cdots \frac{\partial}{\partial t_{k_{n}}} F(t),
$$

then the KDV equations for $F(t)$ are equivalent to a sequence of recursive relations for $n \geq 1$ :

$$
(2 n+1)\left\langle\left\langle\tau_{n} \tau_{0}^{2}\right\rangle\right\rangle=\left\langle\left\langle\tau_{n-1} \tau_{0}\right\rangle\right\rangle\left\langle\left\langle\tau_{0}^{3}\right\rangle\right\rangle+2\left\langle\left\langle\tau_{n-1} \tau_{0}^{2}\right\rangle\right\rangle\left\langle\left\langle\tau_{0}^{2}\right\rangle\right\rangle+\frac{1}{4}\left\langle\left\langle\tau_{n-1} \tau_{0}^{4}\right\rangle\right\rangle .
$$

In [5] the authors give a simple proof of the Witten conjecture by first proving a recursion formula conjectured by Dijkgraaf-Verlinde-Verlinde in [1], and as corollary they are able to give a simple proof of the Witten conjecture by using asymptotic analysis. In this note, we use the method in [3] to prove the recursion formula in [1], therefore the Witten conjecture without using the asymptotic analysis. Combining the coefficients derived in our note and the approach in [3], we can derive more recursion formulas of Hodge integrals.

\footnotetext{
* Received April 23, 2008; accepted for publication June 6, 2008.

†Department of Mathematics, University of California, Los Angeles, CA 90095, USA (chenlin@ math.ucla.edu).

${ }^{\ddagger}$ Department of Mathematics, Harvard University, Cambridge, MA 02138, USA (liyi@math.har vard.edu).

${ }^{\S}$ Center of Mathematical Sciences, Zhejiang University, Hangzhou, China; Department of Mathematics, University of California, Los Angeles, CA 90095, USA (liu@math.ucla.edu; liu@ cms.zju.edu.cn).
} 
2. Localization and the Hurwitz Numbers. Denote by $\mu$ a partition of $d>0$. Let $\overline{\mathcal{M}}_{g, 0}\left(\mathbb{P}^{1}, \mu\right)$ be the moduli space of relative stable morphism to $\mathbb{P}^{1}$, which is a Deligne-Mumford stack of virtual dimension $r=2 g-2+d+l(\mu)$ constructed in [8]. We refer readers to [9] for the property of $\overline{\mathcal{M}}_{g, 0}\left(\mathbb{P}^{1}, \mu\right)$. The $\mathbb{C}^{*}$-action on $\mathbb{P}^{1}$

$$
t \cdot\left[z^{0}: z^{1}\right]=\left[t z^{0}: z^{1}\right],
$$

induces an $\mathbb{C}^{*}$-action on $\overline{\mathcal{M}}_{g, 0}\left(\mathbb{P}^{1}, \mu\right)$. There is a branching morphism

$$
\mathrm{Br}: \overline{\mathcal{M}}_{g, 0}\left(\mathbb{P}^{1}, \mu\right) \longrightarrow \mathbb{P}^{r}
$$

with this action, the branching morphism is $\mathbb{C}^{*}$-equivariant. The Hurwitz numbers can be defined by

$$
H_{g, \mu}:=\int_{\left.\left[\overline{\mathcal{M}}_{g, 0}\left(\mathbb{P}^{1}, \mu\right)\right]\right]^{\mathrm{virt}}} \operatorname{Br}^{*} H^{r}
$$

with the hyperplane class $H \in H^{2}\left(\mathbb{P}^{r} ; \mathbb{Z}\right)$.

2.1. Localization and Hurwitz Numbers. From the localization formula in [9], we have

$$
H_{g, \mu}=(-1)^{k} k ! \widetilde{I}_{g, \mu}^{k},
$$

where $\widetilde{I}_{g, \mu}^{k}$ are the contributions of graphs of $\overline{\mathcal{M}}_{g, 0}\left(\mathbb{P}^{1}, \mu\right)$. Taking $k=0$, it implies the well-known ELSV formula [2]:

$$
H_{g, \mu}=\frac{r !}{|\operatorname{Aut}(\mu)|} \prod_{i=1}^{l(\mu)} \frac{\mu_{i}^{\mu_{i}}}{\mu_{i} !} \int_{\overline{\mathcal{M}}_{g, l(\mu)}} \frac{\Lambda_{g}^{\vee}(1)}{\prod_{i=1}^{l(\mu)}\left(1-\mu_{i} \psi_{i}\right)} .
$$

For $k=1$, it becomes the cut-and-join equation

$$
\begin{aligned}
H_{g, \mu} & =\sum_{\nu \in J(\mu)} I_{1}(\nu) H_{g, \nu}+\sum_{\nu \in C(\mu)} I_{2}(\nu) H_{g-1, \nu} \\
& +\sum_{g_{1}+g_{2}=g} \sum_{\nu^{1} \cup \nu^{2} \in C(\mu)}\left(\begin{array}{c}
r-1 \\
2 g_{1}-2+\left|\nu^{1}\right|+l\left(\nu^{1}\right)
\end{array}\right) I_{3}\left(\nu^{1}, \nu^{2}\right) H_{g_{1}, \nu^{1}} H_{g_{2}, \nu^{2}} .
\end{aligned}
$$

2.2. Notations. In this subsection, we explain some notations appeared in the above subsection. Let $\mu: \mu_{1} \geq \cdots \geq \mu_{n}>0$, and for each positive integer $i$, denote $m_{i}(\mu)$ the number of the integers $i$ appear in $\mu$. Recall the definitions of $J_{\mu}$ and $C_{\mu}$ (see [5] or [7])

$J^{i, j}(\mu)=\left\{\left(\mu_{1}, \cdots, \widehat{\mu}_{i}, \cdots, \widehat{\mu}_{j}, \cdots, \mu_{n}, \mu_{i}+\mu_{j}\right)\right\}, J(\mu)=\cup_{i=1}^{n} \cup_{j=i+1}^{n} J^{i, j}(\mu) ;$

$C^{i, p}(\mu)=\left\{\left(\mu_{1}, \cdots, \widehat{\mu}_{i}, \cdots, \mu_{n}, p, \mu_{i}-p\right)\right\}, C^{i}(\mu)=\cup_{p=1}^{\mu_{i}} C^{i, p}(\mu), C(\mu)=\cup_{i=1}^{n} C^{i}(\mu)$.

If $\nu \in J^{i, j}(\mu)$, then write $\nu:=\mu^{i, j}$, and the $I_{1}(\nu)$ is given by

$$
I_{1}(\nu)=\frac{\mu_{i}+\mu_{j}}{1+\delta_{\mu_{j}}^{\mu_{i}}} m_{\mu_{i}+\mu_{j}}\left(\mu^{i, j}\right) .
$$

For $\nu \in C^{i, p}(\mu)$, then write $\nu=\mu^{i, p}$, and the $I_{2}(\nu)$ is defined by

$$
I_{2}(\nu)=\frac{p\left(\mu_{i}-p\right)}{1+\delta_{\mu_{i}-p}^{p}} m_{p}(\nu)\left(m_{\mu_{i}-p}(\nu)-\delta_{\mu_{i}-p}^{p}\right) .
$$


If $\nu \in C^{i, p}(\mu)$, then let $\nu^{1} \cup \nu^{2}=\nu$, and $I_{3}\left(\nu^{1}, \nu^{2}\right)$ is defined by

$$
I_{3}\left(\nu^{1}, \nu^{2}\right)=\frac{p\left(\mu_{i}-p\right)}{1+\delta_{\mu_{i}-p}^{p}} m_{p}\left(\nu^{1}\right) m_{\mu_{i}-p}\left(\nu^{2}\right) .
$$

Define the formal power series

$$
\Phi(\lambda, p)=\sum_{\mu} \sum_{g \geq 0} H_{g, \mu} \frac{\lambda^{2 g-2+|\mu|+l(\mu)}}{(2 g-2+|\mu|+l(\mu)) !} p_{\mu} .
$$

It is well known that $\Phi(\lambda, p)$ satisfies the following version of cut-and-join equation $[10]$

$$
\frac{\partial \Phi}{\partial \lambda}=\frac{1}{2} \sum_{i . j \geq 1}\left(i j p_{i+j} \frac{\partial^{2} \Phi}{\partial p_{i} \partial p_{j}}+i j p_{i+j} \frac{\partial \Phi}{\partial p_{i}} \frac{\partial \Phi}{\partial p_{j}}+(i+j) p_{i} p_{j} \frac{\partial \Phi}{\partial p_{i+j}}\right)
$$

Define

$$
\Phi_{g, n}(z, p)=\sum_{d \geq 1} \sum_{\mu \vdash d, l(\alpha)=n} \frac{H_{g, \mu}}{r !} p_{\mu} z^{d}
$$

which can be written into the following form by Equation 2.4

$$
\Phi_{g, n}(z ; p)=\frac{1}{n !} \sum_{b_{1}, \cdots, b_{n} \geq 0,0 \leq k \leq g}(-1)^{k}\left\langle\tau_{b_{1}} \cdots \tau_{b_{n}} \lambda_{k}\right\rangle_{g} \prod_{i=1}^{n} \phi_{b_{i}}(z ; p)
$$

where

$$
\phi_{i}(z ; p)=\sum_{m \geq 0} \frac{m^{m+i}}{m !} p_{m} z^{m}, \quad i \geq 0
$$

3. Symmetrization Operator and Rooted Tree Series. In this section, we use the method of Goulden-Jackson-Vakil in [3] to prove the recursion formula, which implies the Witten conjecture/Kontsevich theorem. The proof consists of the following steps: (1) introduce three operators to change the variables as in [3]; (2) compare the leading coefficients of both sides of the cut-and-join equation to derive the recursion formula. Kim-Liu's proof of this recursion formula is via the asymptotic analysis. They write each $\mu_{i}=x_{i} N$ for some $x_{i} \in \mathbb{Q}$ and let $N \in \mathbb{N}$ goes to infinity. The main technic in [5] is the asymptotic estimate of series

$$
\sum_{p=1}^{n} \frac{p^{p+i}}{p !}, \quad \sum_{p+q=n} \frac{p^{p+i+1} q^{q+j+1}}{p ! q !}
$$

for any $i, j \in \mathbb{N}$. The idea here is that by applying the transcendental changing of variable formula in [3], the cut-and-join equation becomes a system of polynomial equalities, which avoid the technical asymptotic estimate.

3.1. Symmetrization Operator. First, we symmetrize $\Phi_{g, n}(z, p)$ by using the linear symmetrization operator $\Xi_{n}$ defined by

$$
\Xi_{n}\left(p_{\alpha} z^{|\alpha|}\right)=\delta_{l(\alpha), n} \sum_{\sigma \in S_{n}} x_{\sigma(1)}^{\alpha_{1}} \cdots x_{\sigma(n)}^{\alpha_{n}} .
$$


The following lemma is elementary, see [3].

Lemma 3.1. For $n, g \geq 0$ and assume $n \geq 3$ if $g=0$, then we have

$$
\begin{aligned}
& \Xi_{n}\left(\Phi_{g, n}(z, p)\right)\left(x_{1}, \cdots, x_{n}\right) \\
& =\frac{1}{n !} \sum_{b_{1}, \cdots, b_{n} \geq 0,0 \leq k \leq g}(-1)^{k}\left\langle\tau_{b_{1}} \cdots \tau_{b_{n}} \lambda_{k}\right\rangle_{g} \sum_{\sigma \in S_{n}} \prod_{i=1}^{n} \phi_{b_{i}}\left(x_{\sigma(i)}\right),
\end{aligned}
$$

where

$$
\phi_{i}(x):=\phi(x ; 1)=\sum_{m \geq 1} \frac{m^{m+i}}{m !} x^{m}
$$

3.2. Rooted Tree Series. The rooted tree series $w(x)$ is introduced in [3]:

$$
w(x)=\sum_{m \geq 1} \frac{m^{m-1}}{m !} x^{m},
$$

which is the unique formal power series solution of the functional equation

$$
w=x e^{w} .
$$

Thus we have

$$
\phi_{i}(x)=\left(x \frac{d}{d x}\right)^{i+1} w(x):=\nabla_{x}^{i+1} w(x)
$$

with $\nabla_{x}:=x \frac{d}{d x}$.

Let $y(x)=\frac{1}{1-w(x)}$ and $y_{j}=y\left(x_{j}\right)$, then $y-1$ is a uniformizer in the ring $\mathbb{Q}[[x]]$. Consider the changing of variables operator $L: \mathbb{Q}[[x]] \rightarrow \mathbb{Q}[[y-1]]$, which send a formal power series in the variable $x$ into a formal power series in the variable $y-1$. In this paper, apply the operator $L$ to formal power series $\phi_{i}(x)$, the formal power series we obtain are in fact polynomials in $y$. We prove this fact in the following lemma.

Lemma 3.2. Denote $w_{j}=w\left(x_{j}\right)$, then

$$
\begin{aligned}
L \nabla_{x_{j}} & =\left(y_{j}^{2}-y_{j}\right) \nabla_{y_{j}} L, \quad L \nabla_{w_{j}}=\left(y_{j}-1\right) \nabla_{y_{j}} L, \\
L\left(\phi_{i}\left(x_{j}\right)\right) & =\left[\left(y_{j}^{2}-y_{j}\right) \nabla_{y_{j}}\right]^{i}\left(y_{j}-1\right), \quad i \geq 0 .
\end{aligned}
$$

Proof. Differentiating the Equation 3.5, we obtain

$$
\nabla_{x_{j}}=\frac{1}{1-w_{j}} \nabla_{w_{j}}
$$

Note that $d y_{j}=y_{j}^{2} d w$, then $\nabla_{w_{j}}=\left(y_{j}-1\right) \nabla_{y_{j}}$ and

$$
L \nabla_{x_{j}}=L\left(\frac{1}{1-w_{j}} \nabla_{w_{j}}\right)=y_{j}\left(y_{j}-1\right) \nabla_{y_{j}} L
$$

The rest identities are left to the readers. 
4. Proof of the Dijkgraaf-Verlinde-Verlinde Conjecture. For $i, j \geq 0$, $i+j \leq n$, let $\underset{i, j}{\stackrel{x}{\Xi}}$ be the mapping, applied to a series in $x_{1}, \cdots, x_{n}$, given by

$$
\underset{i, j}{\Xi} f\left(x_{1}, \cdots, x_{n}\right)=\sum_{\mathcal{R}, \mathcal{S}, \mathcal{T}} f\left(x_{\mathcal{R}}, x_{\mathcal{S}}, x_{\mathcal{T}}\right)
$$

where the summation is over all ordered partitions $(\mathcal{R}, \mathcal{S}, \mathcal{T})$ of $\{1, \cdots, n\}$, where $\mathcal{R}=\left\{x_{r_{1}}, \cdots, x_{r_{i}}\right\}, \mathcal{S}=\left\{x_{s_{1}}, \cdots, x_{s_{j}}\right\}, \mathcal{T}=\left\{x_{t_{1}}, \cdots, x_{t_{n-i-j}}\right\}$ and

$$
\left(x_{\mathcal{R}}, x_{\mathcal{S}}, x_{\mathcal{T}}\right)=\left(x_{r_{1}}, \cdots, x_{r_{i}}, x_{s_{1}}, \cdots, x_{s_{j}}, x_{t_{1}}, \cdots, x_{t_{n-i-j}}\right)
$$

and where $r_{1}<\cdots<r_{i}, s_{1}<\cdots<s_{j}$, and $t_{1}<\cdots<t_{n-i-j}$. The following result gives an expression for the result of applying the symmetrization operator $\Xi_{n}$ to the cut-and-join equation for $\Phi_{g, n}(z, p)$. Denote $\triangle_{y_{j}}:=\left(y_{j}^{2}-y_{j}\right) \nabla_{y_{j}}$. Applying the symmetrization operator $\Xi_{n}$ to the cut-and-join Equation, Goulden-Jackson-Vakil prove the following version of cut-and-join equation [3]

$$
\left(\sum_{i=1}^{n}\left(y_{i}-1\right) \nabla_{y_{i}}+n+2 g-2\right) L \Xi_{n} \Phi_{g, n}\left(y_{1}, \cdots, y_{n}\right)=T_{1}^{\prime}+T_{2}^{\prime}+T_{3}^{\prime}+T_{4}^{\prime},
$$

where

$$
\begin{aligned}
T_{1}^{\prime}= & \left.\frac{1}{2} \sum_{i=1}^{n}\left(\triangle_{y_{i}} \triangle_{y_{n+1}} L \Xi_{n+1} \Phi_{g-1, n+1}\left(y_{1}, \cdots, y_{n+1}\right)\right)\right|_{y_{n+1}=y_{i}}, \\
T_{2}^{\prime}= & \Xi_{1,1} y_{1}^{2} \frac{y_{2}-1}{y_{1}-y_{2}} \triangle_{y_{1}} L \Xi_{n-1} \Phi_{g, n-1}\left(y_{1}, y_{3}, \cdots, y_{n}\right), \\
T_{3}^{\prime}= & \sum_{k=3}^{n} \underset{1, k-1}{\stackrel{y}{\Xi}}\left(\triangle_{y_{1}} L \Xi_{k} \Phi_{0, k}\left(y_{1}, \cdots, y_{k}\right)\right)\left(\triangle_{y_{1}} L \Xi_{n-k+1} \Phi_{g, n-k+1}\left(y_{1}, y_{k+1}, \cdots, y_{n}\right)\right), \\
T_{4}^{\prime}= & \frac{1}{2} \sum_{1 \leq k \leq n, 1 \leq a \leq g-1} \underset{1, k-1}{\stackrel{y}{\Xi}}\left(\triangle_{y_{1}} L \Xi_{k} \Phi_{a, k}\left(y_{1}, \cdots, y_{k}\right)\right) \\
& \cdot\left(\triangle_{y_{1}} L \Xi_{n-k+1} \Phi_{g-a, n-k+1}\left(y_{1}, y_{k+1}, \cdots, y_{n}\right)\right) .
\end{aligned}
$$

4.1. Expansions. We have the following expansion

$$
L\left(\prod_{i=1}^{n} \phi_{b_{i}}\left(x_{\sigma(i)}\right)\right)=\prod_{i=1}^{n}\left(2 b_{i}-1\right) ! ! y_{\sigma(i)}^{2 b_{i}+1}+\text { lower terms. }
$$

From this point, we see that the polynomial $L \Xi_{n} H_{n}^{g}\left(y_{1}, \cdots, y_{n}\right)$ can be written as

$$
L \Xi_{n} \Phi_{g, n}\left(y_{1}, \cdots, y_{n}\right)=\sum_{b_{1}+\cdots+b_{n}=3 g-3+n}\left\langle\tau_{b_{1}} \cdots \tau_{b_{n}}\right\rangle_{g} \prod_{i=1}^{n}\left(2 b_{i}-1\right) ! ! y_{i}^{2 b_{i}+1}+\text { l.t. }
$$

where l.t. denote lower order terms. We write the left hand side of Equation 4.2 by LHS while another side by $\mathrm{RHS}_{1}, \mathrm{RHS}_{2}, \mathrm{RHS}_{3}$ and $\mathrm{RHS}_{4}$, then

$$
\begin{aligned}
\text { LHS } & =\sum_{i=1}^{n} y_{i} \nabla_{y_{i}} \sum_{b_{1}+\cdots+b_{n}=3 g-3+n}\left[\left\langle\tau_{b_{1}} \cdots \tau_{b_{n}}\right\rangle_{g}\left(2 b_{1}-1\right) ! ! \cdots\left(2 b_{n}-1\right) ! !\right] \prod_{l=1}^{n} y_{l}^{2 b_{l}+1}+\text { l.t. } \\
& =\sum_{b_{1}+\cdots+b_{n}=3 g-3+n}\left[\left\langle\tau_{b_{1}} \cdots \tau_{b_{n}}\right\rangle_{g}\left(2 b_{1}-1\right) ! ! \cdots\left(2 b_{n}-1\right) ! !\right] \sum_{i=1}^{n}\left(2 b_{i}+1\right) y_{i} \prod_{l=1}^{n} y_{l}^{2 b_{l}+1}+\text { l.t. }
\end{aligned}
$$




$$
\begin{aligned}
& R H S_{1}=\frac{1}{2} \sum_{b_{1}+\cdots+b_{n+1}=3 g-5+n}\left[\left\langle\tau_{b_{1}} \cdots \tau_{b_{n+1}}\right\rangle_{g-1}\left(2 b_{1}-1\right) ! ! \cdots\left(2 b_{n+1}-1\right) ! !\right] \\
& \left.\cdot \sum_{i=1}^{n}\left(\left(2 b_{i}+1\right)\left(2 b_{n+1}+1\right) y_{i}^{2} y_{n+1}^{2} \prod_{l=1}^{n+1} y_{l}^{2 b_{l}+1}\right)\right|_{y_{i}=y_{n+1}}+\text { l.t. } \\
& R H S_{2}=\underset{1,1}{\Xi}\left(\sum_{b_{1}+b_{3}+\cdots+b_{n}=3 g-4+n}\left[\left(2 b_{1}+1\right) ! !\left(2 b_{3}-1\right) ! ! \cdots\left(2 b_{n}-1\right) ! !\left\langle\tau_{b_{1}} \tau_{b_{3}} \cdots \tau_{b_{n}}\right\rangle_{g}\right]\right. \\
& \left.\sum_{m \geq 0}\left(\frac{y_{2}}{y_{1}}\right)^{m} y_{2} y_{1}^{3} y_{1}^{2 b_{1}+1} \prod_{l=2}^{n} y_{l}^{2 b_{l}+1}\right)+ \text { l.t. } \\
& R H S_{3}=\sum_{k=3}^{n} \underset{1, k-1}{\stackrel{y}{\leftrightarrows}}\left(\sum_{b_{1}+\cdots+b_{k}=k-3}\left(2 b_{1}-1\right) ! ! \cdots\left(2 b_{k}-1\right) ! !\left\langle\tau_{b_{1}} \cdots \tau_{b_{k}}\right\rangle_{0}\left(2 b_{1}+1\right) y_{1}^{2} \prod_{l=1}^{k} y_{l}^{2 b_{l}+1}\right) \\
& \cdot\left(\sum_{\bar{b}_{1}+b_{k+1}+\cdots+b_{n}=3 g-k-2+n}\left[\left(2 \bar{b}_{1}-1\right) ! !\left(2 b_{k+1}-1\right) ! ! \cdots\left(2 b_{n}-1\right) ! !\left\langle\tau_{\bar{b}_{1}} \tau_{b_{k+1}} \cdots \tau_{b_{n}}\right\rangle_{g}\right]\right. \\
& \left.\cdot\left(2 \bar{b}_{1}+1\right) y_{1}^{2} \prod_{l=k+1}^{n} y_{l}^{2 b_{l}+1} y_{1}^{2 \bar{b}_{1}+1}\right)+ \text { l.t. } \\
& R H S_{4}=\frac{1}{2} \sum_{1 \leq k \leq n, 1 \leq a \leq g-1} \underset{1, \frac{y}{\leftrightarrows}}{\stackrel{y}{\leftrightarrows}-1} \\
& \cdot\left(\sum_{b_{1}+\cdots+b_{k}=3 a-3+k}\left(2 b_{1}-1\right) ! ! \cdots\left(2 b_{k}-1\right) ! !\left\langle\tau_{b_{1}} \cdots \tau_{b_{k}}\right\rangle_{a}\left(2 b_{1}+1\right) y_{1}^{2} \prod_{l=1}^{k} y_{l}^{2 b_{l}+1}\right) \\
& \cdot\left(\sum _ { \overline { b } _ { 1 } + b _ { k + 1 } + \cdots + b _ { n } = 3 g - k - 2 + n - 3 a } \left[\left(2 \bar{b}_{1}-1\right) ! !\left(2 b_{k+1}-1\right) ! ! \cdots\left(2 b_{n}-1\right) ! !\right.\right. \\
& \left.\left.\cdot\left\langle\tau_{\bar{b}_{1}} \tau_{b_{k+1}} \cdots \tau_{b_{n}}\right\rangle_{g-a}\right]\left(2 \bar{b}_{1}+1\right) y_{1}^{2} \prod_{l=k+1}^{n} y_{l}^{2 b_{l}+1} y_{1}^{2 \bar{b}_{1}+1}\right)+ \text { l.t. }
\end{aligned}
$$

4.2. Picking Out the Coefficients. Now, we only consider the coefficients of monomial $y_{1}^{2\left(b_{1}+1\right)} y_{2}^{2 b_{2}+1} \cdots y_{n}^{2 b_{n}+1}$ for $b_{1}+\cdots+b_{n}=3 g-3+n$ on both sides of Equation 4.2. By simply calculating, these coefficients are given by

$$
\begin{aligned}
L H S & =\left(2 b_{1}+1\right) ! !\left(2 b_{2}-1\right) ! ! \cdots\left(2 b_{n}-1\right) ! !\left(\left\langle\tau_{b_{1}} \cdots \tau_{b_{n}}\right\rangle_{g}\right. \\
R H S_{1} & =\frac{1}{2} \sum_{a+b=b_{1}-2}(2 a+1) ! !(2 b+1) ! ! \prod_{l=2}^{n}\left(2 b_{l}-1\right) ! !\left\langle\tau_{a} \tau_{b} \tau_{b_{2}} \cdots \tau_{b_{n}}\right\rangle_{g-1} \\
R H S_{2}= & \sum_{l=2}^{n}\left(2\left(b_{1}+b_{l}-1\right)+1\right) ! !\left(2 b_{2}-1\right) ! ! \cdots\left(2 b_{l-1}-1\right) ! !\left(2 b_{l+1}-1\right) ! ! \cdots\left(2 b_{n}-1\right) ! ! \\
& \cdot\left\langle\sigma_{b_{1}+b_{l}-1} \sigma_{b_{2}} \cdots \sigma_{b_{l-1}} \sigma_{b_{l+1}} \cdots \sigma_{b_{n}}\right\rangle_{g} \\
R H S_{3,4} & =\frac{1}{2} \sum_{X \cup Y=S} \sum_{a+b=b_{1}-2} \sum_{g_{1}+g_{2}=g}(2 a+1) ! !(2 b+1) ! ! \prod_{l=2}^{n}\left(2 b_{l}-1\right) ! ! \\
& \cdot\left\langle\tau_{a} \prod_{\alpha \in X} \tau_{\alpha}\right\rangle_{g_{1}}\left\langle\tau_{b} \prod_{\beta \in Y} \tau_{\beta}\right\rangle_{g_{2}},
\end{aligned}
$$


where $S=\left\{b_{2}, \cdots, b_{n}\right\}$. Multiplying the constant $\left(2 b_{2}+1\right) \cdots\left(2 b_{n}+1\right)$, we obtain the recursion formula of Dijkgraaf-Verlinde-Verlinde, which implies the Witten conjecture

$$
\begin{aligned}
\left\langle\widetilde{\tau}_{b_{1}} \prod_{l=2}^{n} \widetilde{\tau}_{b_{l}}\right\rangle_{g}= & \sum_{l=2}^{n}\left(2 b_{l}+1\right)\left\langle\widetilde{\tau}_{b_{1}+b_{l}-1} \prod_{k=2, k \neq l}^{n} \widetilde{\tau}_{b_{k}}\right\rangle_{g}+\frac{1}{2} \sum_{a+b=b_{1}-2}\left\langle\widetilde{\tau}_{a} \widetilde{\tau}_{b} \prod_{l=2}^{n} \widetilde{\tau}_{b_{l}}\right\rangle_{g-1} \\
& \frac{1}{2} \sum_{X \cup Y=\left\{b_{2}, \cdots, b_{n}\right\}}\left\langle\widetilde{\tau}_{\sum_{a+b=b_{1}-2}, g_{1}+g_{2}=g} \prod_{\alpha \in X} \widetilde{\tau}_{\alpha}\right\rangle_{g_{1}}\left\langle\widetilde{\tau}_{b} \prod_{\beta \in Y} \widetilde{\tau}_{\beta}\right\rangle_{g_{2} .} .
\end{aligned}
$$

where $\widetilde{\tau}_{b_{l}}=\left[\left(2 b_{l}+1\right) ! !\right] \tau_{b_{l}}$.

\section{REFERENCES}

[1] R. DiJkgraAf, Intersection Theory, Integrable Hierarchies and Topological Field Theory, New symmetry principles in quantum field theory (Cargse, 1991), 95-158, NATO Adv. Sci. Inst. Ser. B Phys., 295, Plenum, New York, (1992).

[2] T. Ekedahl, S. Lando, M. Shapiro, A. Vainshtein, Hurwitz numbers and intersections on moduli spaces of curves, Invent. Math., 146 (2001), pp. 297-327.

[3] I.P. Goulden, D. M. Jackson And R. Vakil, A short proof of $\lambda_{g}$ conjecture without GromovWitten theory: Hurwize theory and the moduli of curves, preprint: math.AG/0604297.

[4] M. Kazarian, S. Lando, An algebro-geometric proof of Witten's conjecture, J. Amer. Math. Soc., 20 (2007), pp. 1079-1089.

[5] Y.-S. KIM, K. LiU, A simple proof of Witten conjecture through localization, preprint: math.AG/0508384.

[6] M. Kontsevich, Intersection theory on the moduli space of curves and the matrix Airy function, Comm. Math. Phys., 147 (1992), pp. 1-23.

[7] A.M. Li, G. Zhao, Q. Zheng, The number of ramifed coverings of a Riemann surface by Riemann surface, Comm. Math. Phys., 213 (2000), pp. 685-696.

[8] J. LI, Stable Morphisms to singular schemes and relative stable morphisms, J. Diff. Geom., 57 (2001), pp. 509-578.

[9] C.-C. Liu, K. Liu, J. Zhou, A proof of a conjecture of Mariño-Vafa on Hodge Integrals, J. Differential Geom., 65 (2003), pp. 289-340.

[10] C.-C. Liu, K. LiU, J. Zhou, Mariño-Vafa formula and Hodge integral identities, J. Algebric Geom., 15 (2006), pp. 379-398.

[11] M. Mirzakhani, Weil-Petersson volumes and intersection theory on the moduli space of curves, J. Amer. Math. Soc., 20 (2007), pp. 1-23.

[12] A. Okounkov, R. Pandharipande, Gromov-Witten theory, Hurwitz numbers, and Matrix models, I, preprint: math.AG/0101147.

[13] E. Witten, Two-dimensional gravity and intersection theory on moduli space, Surveys in differential geometry (Cambridge, MA, 1990), 243-310, Lehigh Univ., Bethlehem, PA, (1991). 
